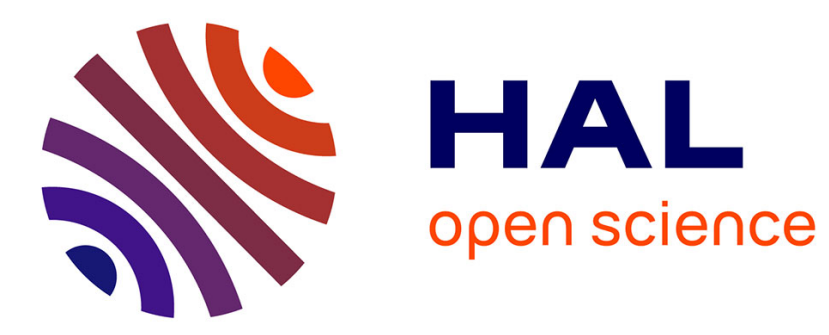

\title{
Télédétection en Méditerranée
}

Lucien Wald, Jean-Marie Monget

\section{To cite this version:}

Lucien Wald, Jean-Marie Monget. Télédétection en Méditerranée. Méditerranée : revue géographique des pays méditerranéens, 1981, I (2 et 3), pp.71-74. hal-00464040

\section{HAL Id: hal-00464040 \\ https://hal-mines-paristech.archives-ouvertes.fr/hal-00464040}

Submitted on 13 Apr 2010

HAL is a multi-disciplinary open access archive for the deposit and dissemination of scientific research documents, whether they are published or not. The documents may come from teaching and research institutions in France or abroad, or from public or private research centers.
L'archive ouverte pluridisciplinaire HAL, est destinée au dépôt et à la diffusion de documents scientifiques de niveau recherche, publiés ou non, émanant des établissements d'enseignement et de recherche français ou étrangers, des laboratoires publics ou privés. 


\title{
Télédétection en Méditerranée
}

\author{
L. WALD * \\ J.M. MONGET *
}

Résumé - Les images de la Méditerranée acquises par les satellites d'observation de la Terre permettent aux Océanographes une nouvelle appréhension, tant spatiale que temporelle, des phénomènes marins. Les deux types de capteurs actuellement opérationnels sont les capteurs passifs visible et infra-rouge. Le domaine visible sert surtout aux Sédimentologues et aux Biologistes, tandis que le domaine infra-rouge est plus adapté à l'étude de la dynamique marine. Ces deux types d'étude ont des répercussions sur l'aide à la pêche.

Abstrat - Satellite imagery gives to Oceanographers the opportunity of a routinely large scale survey of the sea. Visible and infra-red passive sensors are now widely used. Ocean color is studied mainly by Sedimentologists and Biologists, while infra-red imagery is used in Ocean dynamics.

\section{INTRODUCTION}

Si la Télédétection est une technique assez ancienne et déjà bien connue dans le domaine terrestre et côtier, elle est assez nouvelle par contre en Océanographie, où les satellites spécialisés ont fait leur apparition il y a moins de 10 ans.

Si la Télédétection ne concerne que la couche de surface de la mer, elle offre cependant l'avantage d'une vue synoptique et répétitive. Les capteurs opérationnels sont à l'heure actuelle ceux travaillant dans les domaines visibles $(500 \longrightarrow 1000$ nanomètres) et infra-rouge thermique $(10000 \longrightarrow 12000$ nanomètres). Les premiers permettent la connaissance de la couleur de l'océan, tandis que les seconds mesurent la température de la surface de la mer. Il existe d'autres techniques de Télédétection, à partir de satellites utilisant les micro-ondes. Elles permettent l'estimation de :

- l'état de surface de la mer (pente de la mer, hauteur et direction des vagues, vitesse et direction du vent, hauteur de la marée) ;

- la température de surface de la mer.

Ces techniques sont encore expérimentales pour la plupart (SEASAT, NIMBUS - 7), et nous ne nous étendrons pas sur ce sujet.

(*) Centre de Télédétection et d'Analyse des Milieux Naturels (C T A M N), Ecole Nationale Supérieure des Mines de Paris, SOPHIA - ANTIPOLIS, 06560 VALBONNE (France). 


\section{Les satellites}

Le tableau ci-après résume les caractéristiques principales des divers satellites utilisés en Océanographie.

\begin{tabular}{|l|c|c|c|c|}
\hline \multicolumn{1}{|c|}{ Satellite } & $\begin{array}{c}\text { Capteur } \\
\text { visible }\end{array}$ & $\begin{array}{c}\text { Capteur } \\
\text { I. R. }\end{array}$ & $\begin{array}{c}\text { Résolution } \\
\text { spatiale }\end{array}$ & Répétitivité \\
\hline LANDSAT & $\mathrm{X}$ & - & $80 \mathrm{~m}$ & $18 \mathrm{j}$ \\
NIMBUS - 7 & $\mathrm{X}$ & - & $800 \mathrm{~m}$ & 2 à $3 \mathrm{j}$ \\
SPOT & $\mathrm{X}$ & - & $20 \mathrm{~m}$ & $26 \mathrm{j}$ \\
AEM - 1 & $\mathrm{X}$ & $\mathrm{X}$ & $500 \mathrm{~m}$ & 1,5 à $3,5 \mathrm{j}$ \\
Sat. NOAA & $\mathrm{X}$ & $\mathrm{X}$ & $1000 \mathrm{~m}$ & 6 heures \\
\hline
\end{tabular}

Les trois premiers satellites (LANDSAT, NIMBUS-7 et SPOT) disposent de plusieurs canaux dans le domaine visible, alors que les satellites NOAA et AEM-1 n'ont au plus que deux canaux dans ce domaine, et un seul en infra-rouge thermique.

\section{Télédétection dans le visible}

Un capteur visible mesure la luminance provenant de la mer dans le domaine visible. Cette grandeur est composée de la lumière réfléchie par la surface de la mer, ainsi que de la lumière obsorbée par l'océan puis réfléchie vers le haut (rétrodiffusion) par les particules et substances en suspension dans l'eau (Seston, necton, colloides, gelbstoff). L'importance de la composante rétrodiffusée par rapport à la composante réfléchie dépend de la concentration de particules dans la couche superficielle de l'océan, de la longueur d'onde et des positions du satellite et du soleil. C'est ainsi que l'importance relative de la composante réfléchie croîtra avec la longueur d'onde et diminuera lorsque le soleil baissera sur l'horizon, si le satellite a une visée quasi-verticale.

On utilise donc principalement les longueurs d'onde entre 500 et 800 nanomètres pour étudier la couleur de l'océan, c'est-à-dire la turbidité, les transports de sédiments, les concentrations en chlorophylle, etc.

LANDSAT a été utilisé pour la cartographie des transports des sédiments au débouché des fleuves méditerranéens. L'étude des concentrations en seston et necton, quant à elle, s'effectue principalement à l'aide de NIMBUS-7 et est encore dans une phase expérimentale.

Il est évident que cette connaissance de la concentration en seston, maillon du cycle vital, intéresse au plus haut point les pêcheurs.

La mesure de la composante réfléchie de la lumière a été assez peu utilisée. Elle permet l'estimation du coefficient de réflexion de la mer.

Ce coefficient variant en fonction de l'état de la mer, un modèle mathématique utilise la mesure dans le canal $800 \longrightarrow 1000$ nanomètres pour donner une estimation de l'état de surface de la mer et même du vent, puisque l'agitation de la mer dépend de celui-ci. En suivant le même principe, ce canal peut donner également des renseignements sur la pollution par hydrocarbures, puisqu'une tache d'huile a la propriété d'amortir les vagues capillaires responsables de la réflexion vers le capteur, et donc de créer une différence de coefficient de réflexion entre la tache d'huile et la mer agitée environnante.

\section{Télédétection dans l'infra-rouge thermique}

Un capteur infra-rouge thermique mesure la luminance émise par l'océan dans la fenêtre spectrale 10 - 12 microns. Cette luminance dépendant de la température, on obtient ainsi une estimation indirecte de la température de surface de l'eau.

Bien qu'elle soit perturbée par la présence de l'atmosphère, cette température peut être utilisée pour la climatologie marine et météorologique, ainsi que pour la prévision météorologique.

Cette mesure ne concerne malheureusement que le premier mètre d'eau. Cependant, il existe de fortes corrélations entre la distribution spatiale des températures de surface et le champ de courant de la première centaine de mètres. Avoir une image infra-rouge permet d'apprécier la répartition des courants marins. 
La relation observable liant les courants aux températures mesurées par satellite est rarement quantitative, mais cela n'empêche pas l'imagerie infra-rouge d'être d'un grand secours à l'Océanographe dynamicien. L'avènement le plus marquant de ces 'deux dernières années a été la mise en service de capteurs infra-rouges extrêmement performants, montés sur les satellites NOAA et AEM-1. Ils ont montré que le régime général des courants est beaucoup plus compliqué que ce que l'on croyait, et que les courants étaient sujets à des instabilités donnant lieu à des méandres et/ou à des tourbillons tournant dans le sens des aiguilles d'une montre (anticyclonique) ou en sens inverse (cyclonique).

La photo 1 représente une image infra-rouge thermique prise par le satellite TIROS-N le 9 août 1980 à $14 \mathrm{~h} 25 \mathrm{TU}$. On a attribué les teintes noires aux températures les plus chaudes, et les teintes blanches aux plus froides.

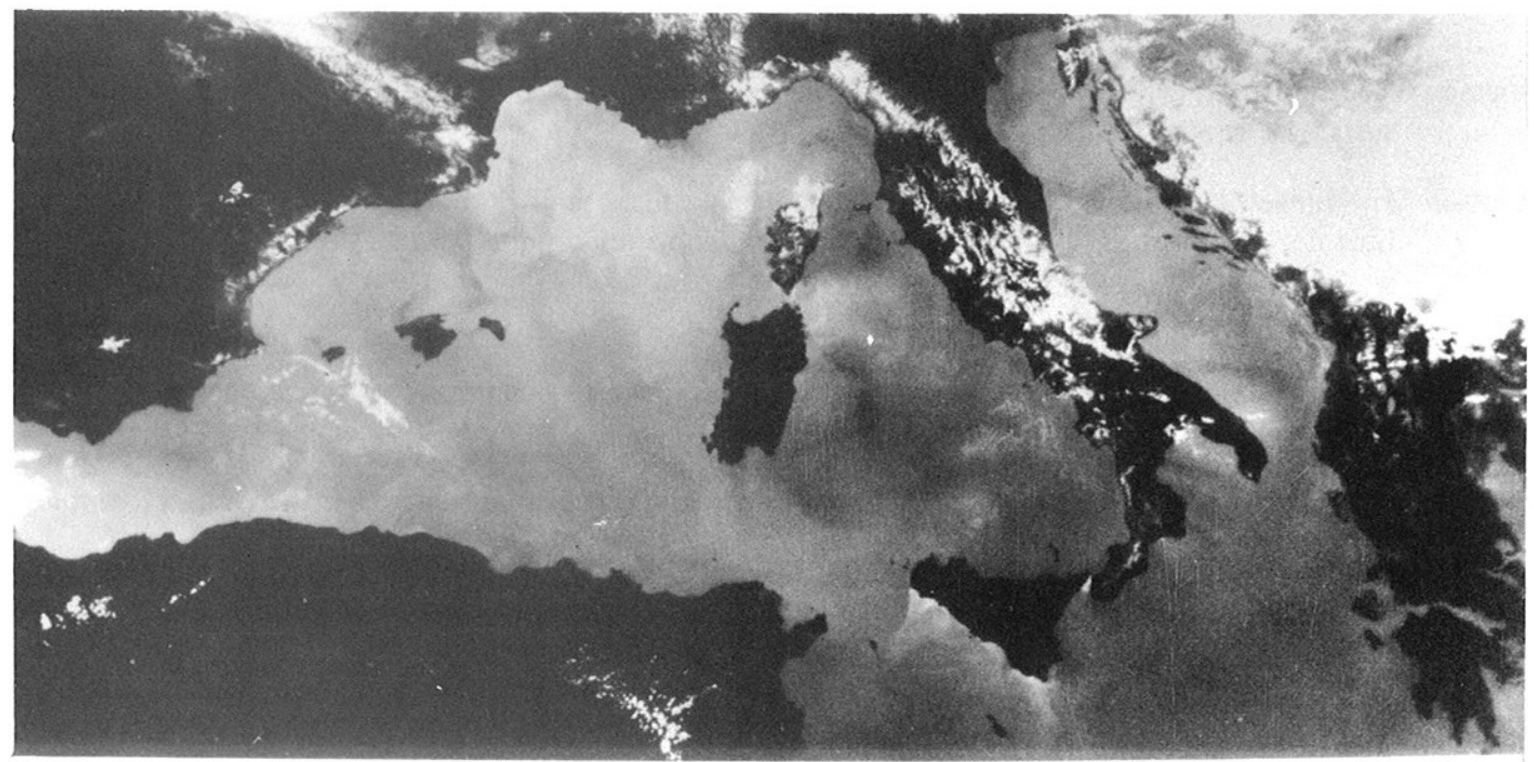

Photo 1 : THERMOGRAPHIE DE LA MEDITERRANEE OCCIDENTALE (d'après le satellite TIROS-N)

Cette photo a été obtenue le $09 / 08 / 80$ à 14 h 25 TU (orbite 9397$)$ dans le canal $4(10,5 \longrightarrow 12,25$ microns). Les teintes les plus sombres correspondent aux températures les plus chaudes, et les teintes les plus claires aux températures les plus froides.

Sur cette photo, on peut voir la veine d'eau d'origine atlantique, qui, entrée en surface à Gibraltar, se déplace le long de la côte algérienne dans une succession de tourbillons et de méandres. Si certains de ces tourbillons peuvent être expliqués par la topographie et la forme des côtes, d'autres ont pour origine des processus physiques encore mal compris. On remarquera le front thermique dit «Nord-Baléares» joignant l'Espagne à la Sardaigne. Ce front est constitué par une série d'indentations, de langues chaudes et froides s'interpénétrant. Il est encore mal connu, mais fait l'objet d'une surveillance attentive. On notera également quelques taches froides accolées à la côte dans le Golfe du Lion et le long de la côte Sud de la Sicile, qui sont la marque de petites remontées d'eau froide (upwellings) dues à l'action du vent sur les eaux de surface. Phénomène curieux : un upwelling est présent dans le détroit de Messine, l'étroitesse même du détroit servant alors d'équivalent à un trait de côte.

\section{CONCLUSION}

Nous avons présenté les principaux visages de l'Océanographie spatiale, en nous cantonnant aux capteurs opérationnels et éprouvés.

Le domaine visible est surtout utilisé pour les études des transports estuariens ou pour la connaissance des concentrations en chlorophylle, phyto et zoo-plancton des eaux plus au large.

Une autre caractéristique, peu utilisée, de ce domaine spectral, est de permettre la connaissance au travers d'un modèle mathématique de l'état d'agitation de la mer, connaissance ayant des répercussions sur la détection de la pollution et de la dynamique marine. 
L'infra-rouge thermique est surtout utilisé en Océanographie dynamique, pour la cartographie et le suivi des fronts thermiques.

Il convient d'insister sur le fait que ces deux techniques ont des répercussions importantes sur la gestion rationnelle des zones de pêche.

Le visible permet de mesurer les concentrations en plancton qui vont nourrir les poissons, tandis que l'infra-rouge permet la détection des fronts thermiques qui sont souvent associés à des mouvements verticaux locaux et à des concentrations importantes de substances organiques.

\section{BIBLIOGRAPHIE}

Albuisson M., Nihous G., Wald L., (1979) : Etude de la cartographie de la couleur et de la température de la surface de la mer. Convention CNEXO 78/1814.

Albuisson M., Monget J. M., (1978) : Méthodes et moyens utilisés pour la mise en forme de données de satellites de télédétection : application à la cartographie thermique. Publ. CNEXO, Actes de colloque $\mathrm{n}^{\circ} 5$, pp. 181-202.

Castaing P., Allen G. P., Houdart M., Moign Y., (1979) : Etude par télédétection de la dispersion en mer des eaux estuariennes issues de la Gironde et du Pertuis de Maumusson. Oceanologica Acta, 2, 4, pp. 459-468.

Millot C., Wald L., (1980) : The effect of Mistral wind on the Ligurian current near Provence. Oceanologica Acta, 3, 4, pp. 399-402.

Morel A., Prieur L., (1978) : Mesures par télédétection de la teneur en chlorophylle. Possibilités et limites des méthodes. Publ. CNEXO, Actes de colloque n 5, pp. 67-92.

Philippe M.,(1980) : Fronts thermiques en Méditerranée, d'après les données du radiomètre du satellite NOAA-5. C. R. Acad. Sc. Paris, t. 291, série B, 43.

Wald L., Nihous G., (1980) : Ligurian sea : annual variation of the sea surface thermal structure as detected by satellite NOAA -5. Oceanologica Acta, 3, 4, pp. 465-470. 